\title{
Hole and Protonic Polarons in Perovskites
}

\author{
Artur Braun*a, Qianli Chen ${ }^{\star b}$, and Arthur Yelon ${ }^{* \mathrm{c}}$
}

\begin{abstract}
Electric charge transport is an essential process for all electrical and electrochemical energy systems, including inanimate and animate matter. In this issue on materials for energy conversion, we compare and discuss the role of electron holes and protons as charge carriers in solids. Specifically we outline how the temperature or thermal bath affect the charge carrier concentration and mobility for some metal oxides with the perovskite structure. The frequent observation that the conductivity becomes independent of the activation energy at the isokinetic temperature, known as the Meyer-Neldel rule, is an important aspect of our interpretation of the physical mechanism of conduction by polaron hopping.
\end{abstract}

Keywords: Electrochemistry · Energy storage $\cdot$ Hydrogen $\cdot$ Meyer-Neldel rule $\cdot$ Polaron $\cdot$ Proton

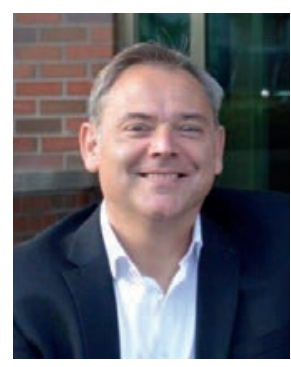

Artur Braun is a Berkeley-trained physicist from RWTH Aachen and holds a doctor of science degree in electrochemistry from ETH Zürich. Dr. Braun is an expert on operando and in situ analyses of materials and electrochemical energy systems with synchrotron X-ray spectroscopy and neutron scattering. He is Marie Curie Fellow and has chaired the Materials Research Society Spring Meeting 2015 in San Francisco. Braun works currently at Empa. He has authored over 150 publications and 4 graduate level textbooks on energy systems. Foreign Policy magazine listed Dr. Artur Braun and his associates as Leading Global Thinkers of 2014 for their research on solar energy.

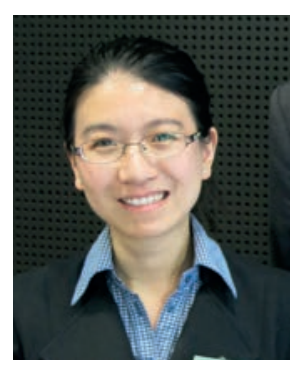

Qianli Chen is Assistant Professor of materials science and engineering at University of Michigan - Shanghai Jiao Tong University Joint Institute. She completed her doctoral thesis with Artur Braun at Empa and Joel Mesot at ETH Zürich. She holds academic degrees from ETHZurich, KTH, Stockholm, and Southeast University, Nanjing. She was an Alexander von Humboldt post-doctoral research fellow at the Max Planck Institute for Polymer Research. Her research focuses on the charge carrier behavior in solids, including the transport and distribution of ions and electrons. Dr. Chen is winner of Young Scientist Prize of the Swiss Neutron Scattering Society in 2014.

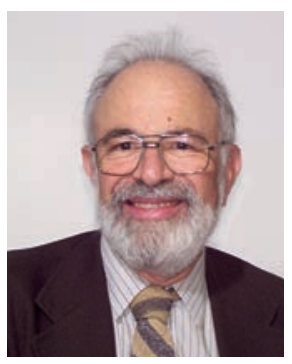

Arthur Yelon is Professor Emeritus of Engineering Physics at Polytechnique Montréal. He received his academic degrees in Physics from Cornell University and Case Institute of Technology (now part of Case Western Reserve University). He held positions at IBM T.J. Watson Research Center, at CNRS Grenoble, and at Yale University before joining Polytechnique Montréal. His research activities over his career have involved thin films and surfaces, amorphous and crystalline semiconductors, magnetism, electron tunnelling, and most recently, high activation energy kinetics, an aspect of the present publication.

\section{Introduction}

Availability of electric energy and power is essential for all electric and electronic devices. Our daily life in the $21^{\text {st }}$ century is increasingly accompanied by such devices: handheld gadgets like cell phones and cameras, residential appliances and entertainment centers, mobility equipment like electric vehicles, as well as renewable power plants which convert solar and wind energy. The principal electric charge carriers in this technological context are the negatively charged electrons. However, cations such as lithium in batteries and protons in fuel cells and electrolyzers play an essential role in electric energy storage and conversion.

Electrochemical energy conversion processes are not subject to the limiting thermodynamic efficiency $\eta$ associated with Carnot machines, ${ }^{[1]}$ which derive their power from two thermodynamic baths, one at a high temperature, $\mathrm{T}_{h}$, and the other at a low temperature, $T_{1}: \eta \leq 1-T_{1} / T_{h}$. The efficiency of an electric machine is determined by $\eta=W / Q_{i n}$, where $W$ is the work provided by the electromotive force, and $\mathrm{Q}_{\mathrm{in}}$ is the enthalpy of the underlying electrochemical reaction. However, this does not mean that temperature is an irrelevant parameter in electrochemical processes, in particular, and in electrical phenomena, in general. The electric resistivity, which causes losses, limiting $\eta$, can be a strong function of the temperature, irrespective of which electric conductors are considered.

Superconductivity, a zero resistivity state, occurs only at extremely low temperatures (for some metals) and high-temperature superconductivity occurs at around $140 \mathrm{~K}$ (for some metal oxides

\footnotetext{
${ }^{*}$ Correspondence: Dr. A. Braun ${ }^{a}$ E-mail: artur.braun@alumni.ethz.ch; Prof. Q. Chen ${ }^{b}$, E-mail: qianli.chen@sjtu.edu.cn; Prof. em. A. Yelonc, E-mail: arthur.yelon@polymtl.ca ${ }^{a}$ Empa. Swiss Federal Laboratories for Materials Science and Technology, Überlandstrasse 129, CH-8600 Dübendorf, Switzerland; bUniversity of Michigan - Shanghai Jiao Tong University Joint Institute, 800 Dong Chuan Road, Shanghai, 200240, China; 'Département de Génie Physique, Polytechnique Montréal, CP 6079, Succursale C-V, Montréal, QC H3C 3A7, Canada, and Réseau Québecois sur des Matériaux de Pointe (RQMP)
} 
for example, with the perovskite structure). The technological goal - for economic reasons and for reasons of sustainable development ${ }^{[2]}-$ is to have superconductivity at ambient conditions with zero energy input. For the various classes of materials (conductors, semiconductors, and insulators) there exist particular conductivity models. Inspired by the observation of electronic superconductivity, researchers have begun to search for materials with ionic superconductivity, including superprotonic conductors. ${ }^{[3]}$

Here, we present a model of protonic conductivity and hole conductivity in some perovskites which can be employed in solid oxide fuel cell cathodes and electrolytes, based, in part, on comparison with the better understood electronic conductivity, and, in part, on measurements of the physical properties of these materials. One aspect of the latter is the investigation of the temperature dependence of conductivity, which, for semiconductors, is a kinetic process, leading to Arrhenius behavior,

$$
\sigma(\mathrm{T})=\sigma_{0} \cdot \mathrm{e}^{\left(\frac{-\mathrm{E}_{\mathrm{a}}}{\mathrm{k}_{\mathrm{B}} \cdot \mathrm{T}}\right)}
$$

In Eqn. (1), $\sigma(\mathrm{T})$ is the conductivity at temperature, $\mathrm{T}, \mathrm{E}$ is the activation energy, $\mathrm{k}_{\mathrm{B}}$ is Boltzmann's constant, and $\sigma_{\mathrm{o}}$ is an experimental prefactor. The electric conductivity increases considerably with increasing temperature. While low activation energy kinetics has been relatively well understood since the $19^{\text {th }}$ century, kinetics at high activation energies (the case for conductivity of perovskites) is less well known, and very much less well understood.

\section{Electronic and Protonic Conductivity of Perovskites}

\subsection{Hole Conductivity}

As an example, Fig. 1 shows the electronic conductivity of an iron perovskite $\mathrm{La}_{0.9} \mathrm{Sr}_{0.1} \mathrm{FeO}_{3}$ (LSF, a material which can be used as solid oxide fuel cell cathode) at temperatures ranging from 273 $\mathrm{K}$ to $1150 \mathrm{~K}$ (single crystal slab and sintered polycrystalline bar measured in air with 4-point DC method). For many metal oxides, including those with perovskite structure, such as LSF, the electronic conductivity follows a typical polaron transport mechanism. ${ }^{[4-9]}$ Briefly, a polaron is a quasi-particle, which arises as a collective excitation by an electric charge, typically an electron, which is dressed in a crystal lattice and sources its energy from the thermodynamic bath of lattice vibrations.

The phonons of the lattice couple to the charged particle and form a polaron. ${ }^{[10]}$ Phenomenologically this is observed as the exponential increase of the conductivity with temperature, with large activation energy and small conductivity prefactor, which give a hint about the polaronic nature of the conductivity. In an Arrhenius plot, the conductivity follows a straight line.

The electronic conductivity,

$$
\sigma(T)=\frac{\sigma_{0}}{T} \cdot e^{\left(\frac{-E_{a}}{k_{B} \cdot T}\right)}
$$

for the material in Fig. 1 comes from $O 2 p$ ligand electron holes $h^{+}$as charge carriers, which originate from the spectroscopically equivalent $\mathrm{Fe}^{4+}$ in the material. The relative hole concentration and thus charge carrier concentration is $n=\left[\mathrm{Fe}^{4+}\right] /\left(\left[\mathrm{Fe}^{4+}\right]+\left[\mathrm{Fe}^{3+}\right]\right)$. Specifically, it turns out that these holes $h^{+}$have $\mathrm{e}_{\mathrm{g}} \uparrow \operatorname{spin}$ up, orbital symmetry and are located in the valence band (VB) in closer proximity to the Fermi level than the electrons $e^{-}$. Holes and electrons can be experimentally detected and verified using oxygen ligand X-ray spectroscopy (NEXAFS, near-edge X-ray absorption fine structure spectroscopy $\left.{ }^{[13]}\right)$. The nearby electronic states with spin down $\mathrm{t}_{2 \mathrm{~g}} \downarrow$ and $\mathrm{e}_{\mathrm{g}} \uparrow$ orbital symmetry can be identified in the NEXAFS as a spectroscopic doublet. The experimental data, the empirical observables so-to-speak, are the NEXAFS spectra and the 4-point DC conductivities. From the stoichiometry, we can determine the charge carrier concentration $n$. Fig. 2 shows how the conductivity scales linearly in the log-plot with the hole concentration for the $\mathrm{La}_{(1-\mathrm{x})} \mathrm{Sr}_{\mathrm{x}} \mathrm{FeO}_{3}$ series with $\mathrm{x}=0,0.20$ and 0.40 .

It has been shown empirically ${ }^{[14,15]}$ that the electronic conductivity $\sigma$ scales exponentially with the relative spectral weight $S$ of these hole states in the NEXAFS spectra, $S=\mathrm{e}_{\mathrm{g}} \uparrow /\left(\mathrm{t}_{2 \mathrm{~g}} \downarrow+\mathrm{e}_{\mathrm{g}} \downarrow\right)$ :

$$
\sigma \sim \sigma^{\prime} \cdot e^{\mathcal{H} \cdot n}
$$

We find from a least squares fit of the experimental data in ref. [14] that $\mathcal{H}=7.88$ (see also later in Table 1), and more specifically $\sigma=0.0185 \cdot \exp (7.88 \cdot n)$. For the charge carrier concentration $n$, we can assume a relative number $0 \leq n \leq 1$, for example $n=1 / 2$ ( 0.52 is a realistic value for $\mathrm{La}_{0.5} \mathrm{Sr}_{0.5} \mathrm{FeO}_{3}$ according to ref. [14]). The relative spectral weight of holes, $S(n)$, is a linear function in the dimensionless $n$ :

$$
S(n)=S_{0}+a \cdot n
$$

For the samples that we had investigated, ${ }^{[14]} S_{0}$ was 0 . These experiments were carried out at ambient temperature, near $300 \mathrm{~K}$. Here, $n$ is the aforementioned relative charge carrier (hole $h^{+}$) con-
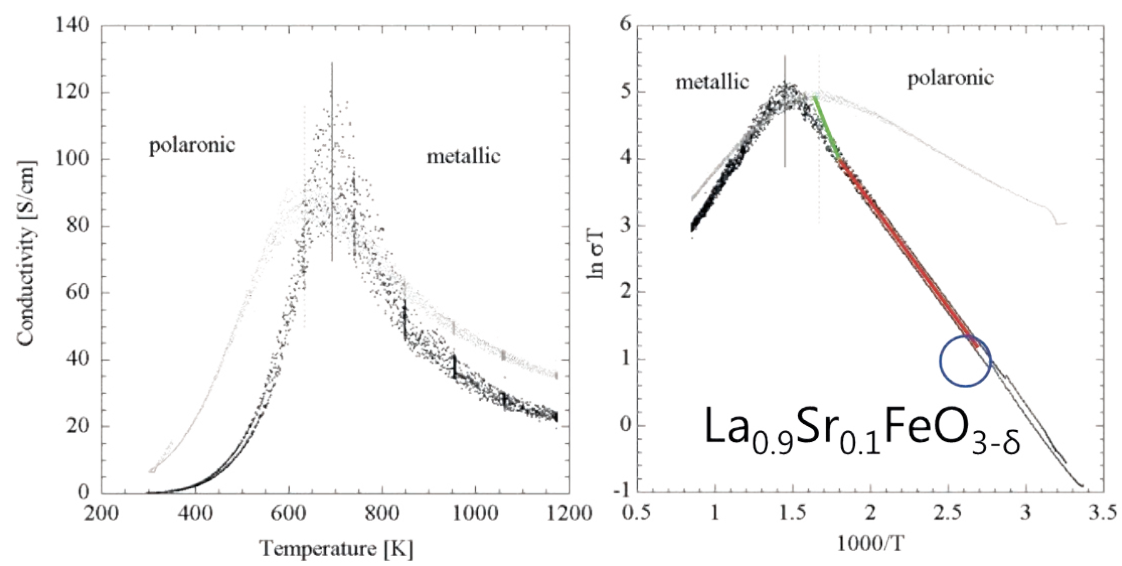

Fig. 1. The 4-point DC electric conductivity of LSF, from $300 \mathrm{~K}$ to $1200 \mathrm{~K}$ in air in linear coordinates (left) and in Arrhenius coordinates (right) (compare ref. $[11,12])$. The region of exponential increase of conductivity is indicative of the polaron-based conductivity. The light points were recorded from a sintered slab. The dark points were recorded from a cylinder-shaped single crystal. Colored lines indicate small changes in activation energy ${ }^{[10]}$ due to temperature activated changes in the electronic structure of the material. 


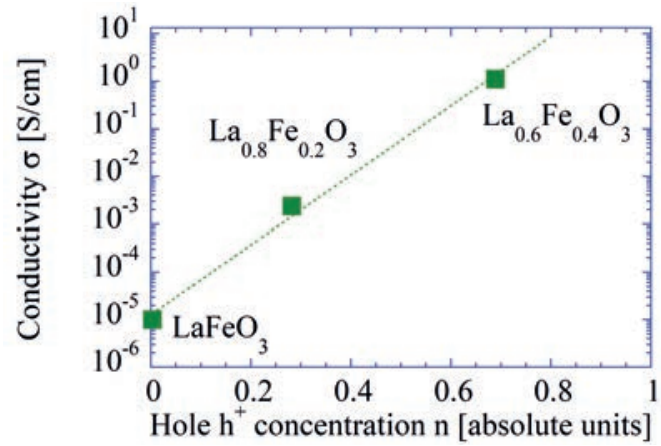

Fig. 2. The 4-point DC electric conductivity of LSF, $\mathrm{La}_{(1-x)} \mathrm{Sr}_{\mathrm{x}} \mathrm{FeO}_{3}$ for $\mathrm{x}=$ $0.00,0.20$ and 0.40 at $300 \mathrm{~K}^{[11,12]}$ as a function of the hole $h^{+}$charge carrier concentration $n$ in a logarithmic plot. The dotted line is a least squares fit to the three data points.

centration and $\mathcal{H}$ some, yet to be defined, dimensionless parameter. The observation that the change of $\sigma$ is exponential, whereas the variation of the hole concentration and the spectral changes are linear, suggests that hole concentration alone cannot account for the entire conductivity variation. To the best of the authors' knowledge, no physical explanation has yet been proposed for the meaning of the quantity $\mathcal{H}$. We shall propose a model, which yields $\mathcal{H}$, below. We discuss the relationship between $\sigma_{o}$ and $\sigma$, further below.

\subsection{Protonic Conductivity}

In the ceramic proton conductors, relevant for solid oxide fuel cells and electrolyzers, we observe similar behavior for the proton conductivity. At low temperatures, the protons are localized as structural ions in hydroxyl groups in the metal oxide (compare refs. $[3,16,17])$. At elevated temperatures, the hydroxyl bonds break and the protons are liberated as charge carriers. ${ }^{[18]}$ The proton conductivity shown in an Arrhenius plot, as in Fig. 3 follows a straight line, from which we obtain large activation energies and small conductivity prefactors. This phenomenological similarity for electronic conductivity and proton conductivity raises the question whether the microscopic physical mechanisms are essentially the same for the conductivity of electrons, electron holes, and ions. Indeed, it is difficult to imagine that holes would act as polaronic carriers, whereas protons would not. Their different size and mass may lead to different local energy minima, but they are both positively charged and thus have the same type of Coulomb interaction with the lattice, being attracted to negatively charged lattice ions, and repelled by positively charged lattice ions. Further, we have found experimental evidence that the protons in ceramic proton conductors follow polaron behavior, ${ }^{[19]}$ as had previously been hypothesized.[20]

Fig. 3 shows an example of the diffusivity of protons in a ceramic pellet of $\mathrm{BaZr}_{0.9} \mathrm{Y}_{0.1} \mathrm{O}_{3-\delta}$, in an Arrhenius plot, which allows for a linear least square fit over particular temperature ranges. We have obtained the raw data ${ }^{[21,22]}$ by conventional impedance spectroscopy (IS), an electroanalytical method, and by quasi-elastic neutron scattering (QENS), which probes the diffusivity, specifically of protons, at the molecular scale. QENS yields an activation energy, for the localized proton in an $\mathrm{OH}^{-}$ structure to rotate around the $\mathrm{O}^{2-}$ ion, of $40 \mathrm{meV}$. This mode does not constitute any effective proton transport suitable for a solid electrolyte. From IS it was found that the activation energy for protons $\mathrm{H}^{+}$to diffuse in the bulk is $460 \mathrm{meV}$, whereas it is $1210 \mathrm{meV}$ for diffusion over grain boundaries. With increasing temperature however, diffusivity and thus conductivity of $\mathrm{O}^{2-}$ ions sets in. Note that impedance spectroscopy, unlike QENS, cannot distinguish between oxygen diffusivity and proton diffusivity.

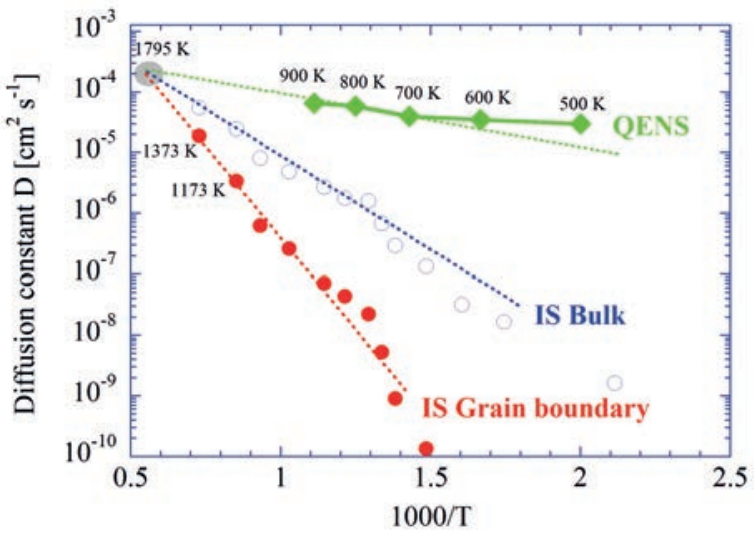

Fig. 3. The diffusivity $\mathrm{D}$ of protons in a compacted $\mathrm{BaZr} \mathrm{r}_{0.9} \mathrm{Y}_{0.1} \mathrm{O}_{3-\delta}$ pellet measured at temperatures $373 \mathrm{~K}<\mathrm{T}<973 \mathrm{~K}$ with quasi-elastic neutron scattering (QENS, green filled symbols) and with impedance spectroscopy (IS, red filled squares deconvoluted for grain boundaries; open blue triangles deconvoluted for the bulk conductivity contribution). The dashed lines are extrapolations to high temperature which meet at $1795 \mathrm{~K}$ and a diffusion constant of $2 \cdot 10^{-4} \mathrm{~cm}^{2} / \mathrm{s}$. After ref. [21].

\section{High Activation Energy Kinetics, Multi-excitation Entropy, and Isokinetic Temperature}

In addition to the electric conductivity of solids, many phenomena and processes obey the Arrhenius equation, which is a generalization of Eqn. (1), with prefactors appropriate for the phenomenon in question. In 1937, Eyring showed that it is the free energy of activation, and not only the activation energy, which determines the rate $k$ for a chemical reaction, which obeys the equation: ${ }^{[23]}$

$$
\mathrm{k}=\frac{\mathrm{k}_{\mathrm{B}} \cdot \mathrm{T}}{\mathrm{h}} \cdot \mathrm{e}^{\left(-\frac{\Delta \mathrm{G}}{\mathrm{k}_{\mathrm{B}} \cdot \mathrm{T}}\right)}=\frac{\mathrm{k}_{\mathrm{B}} \cdot \mathrm{T}}{\mathrm{h}} \cdot \mathrm{e}^{\left(\frac{\Delta \mathrm{S}}{\mathrm{k}_{\mathrm{B}}}\right)} \cdot \mathrm{e}^{\left(-\frac{\Delta \mathrm{H}}{\mathrm{k}_{\mathrm{B}} \cdot \mathrm{T}}\right)}
$$

In Eqn. (5), which implies the relation $\Delta H=\Delta \mathrm{G}+\mathrm{T} \Delta \mathrm{S}, h$ is Planck's constant (not to be mistaken for the positive charge of the hole $h^{+}$), and $\Delta \mathrm{G}, \Delta \mathrm{S}$, and $\Delta \mathrm{H}$ are the free energy, entropy, and enthalpy changes in going from the initial state to the transition state of the barrier. It is clear that the prefactor, $\sigma_{o}$ of Eqn. (1) should also include an entropy term, as in Eqn. (5), even if this term cannot be directly obtained from an Arrhenius diagram. It is also clear that $\Delta \mathrm{H}=E_{\mathrm{a}}$ for incompressible solids, as volumes are essentially independent of pressure, for typical experimental conditions.

From a microscopic point of view, it is easy to understand that when $E_{a}$ is of the order of the energies of excitations of the solid, phonons or local vibrations, the right hand side of Eqn. (1), the final exponential of Eqn. (5), $\exp \left(-\Delta \mathrm{H} / \mathrm{k}_{\mathrm{B}} \cdot \mathrm{T}\right)$ represents the probability of finding an excitation of this energy. Less energetic excitations have no effect on overcoming the barrier, and those that are more energetic are so rare that they practically do not contribute to charge transport. What happens when $E_{a}$ is large compared to excitation energies, and large compared to the thermal energy of the surrounding bath, $\mathrm{k}_{\mathrm{B}} \cdot \mathrm{T}_{\mathrm{m}}\left(\mathrm{T}_{\mathrm{m}}\right.$ is the temperature at which the specimen is measured), is less obvious.

A number of groups pointed out in the 1980s that, in this case, a fluctuation providing multiple excitations is necessary in order to obtain the energy to overcome the activation barrier. ${ }^{[24]}$ The rationale is similar to the mechanism for the catalytic decomposition of hydrocarbons on surfaces originally proposed 1925 by Constable. ${ }^{[25]}$ There, the catalytic action depends on the attempt frequency of atoms and their mechanical impact with the molecules.

Combining increasing numbers of excitations implies that the entropy, $\Delta S$ in the free energy of activation includes a contribution $\Delta \mathrm{S}_{\mathrm{M}}$, which we call multi-excitation entropy (MEE), and which increases with $E_{a}$ or with $\Delta \mathrm{H}: \Delta \mathrm{S}_{\mathrm{M}} / E_{\mathrm{a}}>0$, and $\Delta \mathrm{S}_{\mathrm{M}} / \Delta \mathrm{H}>0$. 
It has been shown theoretically ${ }^{[24,26]}$ that, if all of the excitations have the same energy, as for the Einstein model (this is approximately correct for optical phonons and molecular vibrations), $\Delta \mathrm{S}_{\mathrm{M}}$ is proportional to $E_{\mathrm{a}}$ :

$$
\Delta S_{M}=\frac{E_{a}}{T_{i s o}}
$$

and $\mathrm{T}_{\text {iso }}$ is the isokinetic temperature, as will be further explained below. In general, there is strong evidence that $\Delta \mathrm{S}_{\mathrm{M}}$ is proportional to $(\Delta \mathrm{H})^{\alpha}$, where $\alpha$ ranges between $1 / 2$ and 1 .

Taking account of the possibility of an entropy term in addition to $\Delta \mathrm{S}_{\mathrm{M}}$, independent of $\Delta \mathrm{H}$, combining Eqns (5) and (6) leads to the prediction that $\mathrm{k}$ is independent of $\Delta \mathrm{H}$ (isokinetic) at $\mathrm{T}_{\text {iso }}$. This effect has been experimentally observed for a wide range of phenomena, and is known, synonymously, as the isokinetic law, the Meyer-Neldel rule (MNR), since it was reported by Meyer and Neldel ${ }^{[27]}$ for the conductivity of 'disordered' materials, and the compensation law, since the term proportional to $E_{\mathrm{a}}$ in Eqn. (6) partially 'compensates' for the decrease in $\sigma$ with increasing $E_{\text {a }}$ in Eqn. (1). We express this by the relation

$$
\ln \sigma_{0}=\ln \sigma_{00}+\frac{E_{a}}{k_{B} \cdot T_{\text {iso }}}
$$

A graphic example of the behavior, which may be described by relations like Eqn. (7) is provided by the proton conductivity data in Fig. 3. When we extrapolate the trend of the diffusion constants in Fig. 3 to higher temperatures, the lines coincide at 1795 $\mathrm{K}$. This is the isothermal temperature $\mathrm{T}_{\text {iso }}$, where the diffusion constant is the same, i.e. $D_{00}=2 \cdot 10^{-4} \mathrm{~cm}^{2} / \mathrm{s}$ irrespective of the specific origin of the diffusion. While $\mathrm{T}_{\text {iso }}$ of $1795 \mathrm{~K}$ is comparatively high, it still yields a reasonable value for a thermal energy of $\mathrm{k}_{\mathrm{B}} \cdot \mathrm{T}_{\text {iso }}=$ $155 \mathrm{meV}$ (see Table 1).

Following the theoretical work of Emin ${ }^{[28,29]}$ it was pointed out $^{[30]}$ that we should expect for the isothermal temperature the relation

$$
\mathrm{k}_{\mathrm{B}} \mathrm{T}_{\text {iso }}=\frac{\mathrm{h} v}{\ln \kappa}
$$

where $h v$ is the energy of the excitations which are combined to overcome the activation barrier, and $\kappa$ is a coupling constant, given by the coupling energy divided by the excitation energy. In particular, for polarons we write

$$
\kappa=\frac{E_{\mathrm{b}}}{\mathrm{h} v}
$$

where $E_{\mathrm{b}}$ is the polaron binding energy.

From experiment, $\ln \kappa$ is always between $1 / 2$ and 2 . Then, a $T_{\text {iso }}$ of $1795 \mathrm{~K}$ corresponds to lattice vibrations with wavenumber between 623 and $2492 \mathrm{~cm}^{-1}$, for $\ln \kappa$ between $1 / 2$ and 2 . When we look into this range for the vibration modes for the $\mathrm{Y}$-doped $\mathrm{BaZrO}_{3}$ we find the material has a strong $\mathrm{Y}-\mathrm{O}-(\mathrm{Y}, \mathrm{Zr})$ tilting motion at about $740 \mathrm{~cm}^{-1}(\ln \kappa=0.59){ }^{[31]}$
Eqns (8) and (9) permit us to understand the observation, for a number of material systems, of a correlation between $\sigma_{\text {oо }}$ and $\mathrm{T}_{\text {iso }}{ }^{[32,33]}$ For materials for which the conductivity is for example impeded by neutral traps, we expect, ${ }^{[34]}$ and find, that the conductivity increases as $\mathrm{T}_{\text {iso }}$ decreases, since strong electron-phonon coupling implies a large coupling constant $\kappa$, low $\mathrm{T}_{\text {iso }}$, and high $\sigma$. For polaronic conductors, we expect ${ }^{[34]}$ and observe the opposite, since strong binding leads to low conductivity. This is the case for amorphous chalcogenides, ${ }^{[35]}$ which are known to be polaronic, and for proton conduction in minerals, ${ }^{[36]}$ which has led to the conclusion $^{[37]}$ that this is also a polaron effect. Thus, observation and understanding of MNR support our suggestion that proton conduction in perovskites should also be polaronic.

\section{Discussion}

\subsection{Hole Conductivity}

Following the discussion above, we assume that conductivity in LSF is due to holes (with positive charge $\mathrm{h}^{+}$as opposed to $\mathrm{e}^{-}$), and treat the holes as lattice polarons (small polarons). ${ }^{[38]}$

Then,

$$
\sigma(\mathrm{T})=n_{\mathrm{h}+}(\mathrm{T}) \cdot \mathrm{h}^{+} \cdot \mu(\mathrm{T})
$$

with $n_{\mathrm{h}+}(\mathrm{T})$ the concentration of carriers, and $\mu(\mathrm{T})$, their mobility. Then $\sigma_{o o}$ in Eqn. (7) must be given by $n_{o} \cdot h^{+} \cdot \mu_{o}$, since both $n_{\mathrm{p}}$ and $\mu$ may be activated. The concentration of electron holes is

$$
\mathrm{n}_{\mathrm{h}}+(\mathrm{T})=\mathrm{p}_{0} \cdot \mathrm{e}^{\frac{-\mathrm{E}_{\mathrm{g}}}{\mathrm{k}_{\mathrm{B}} \mathrm{T}}}
$$

where we define

$$
E_{g}=E_{F}-E_{V}
$$

In Eqn. (12), $E_{\mathrm{V}}$ is the maximum of the valence band $\mathrm{VB}$, and $E_{F}$ is the Fermi level (and $E_{g}$ is thus, not the band gap energy). As discussed below, we can consider $E_{\mathrm{g}}$ as a function of charge carrier concentration, and thus of stoichiometry,

$$
E_{g}=E_{g}^{0}-E_{g}^{\prime} \cdot n
$$

with $n$ as defined for Eqns (3) and (4), and $0 \leq n \leq 1$. Band gap energies in $\mathrm{La}_{(1-\mathrm{x})} \mathrm{Sr}_{\mathrm{x}} \mathrm{FeO}_{3}$ are reported to range from $330 \mathrm{meV}$ to $50 \mathrm{meV}$ for $\mathrm{x}=0.15^{\mathrm{x}}$ to $0.9 .{ }^{[39]}$ For the further discussion, we refer to the data for the holes $\mathrm{h}^{+}$listed in Table 1 .

At high temperature, the movement of small polarons is a ran-

\begin{tabular}{|c|c|c|c|c|c|c|c|c|}
\hline Type & $\mathcal{H}$ & $\mathbf{n}$ & $\begin{array}{c}\mathbf{T}_{\mathbf{m}} \\
{[\mathrm{K}]}\end{array}$ & $\begin{array}{c}\mathbf{k}_{\mathbf{B}} \mathbf{T}_{\mathbf{m}} \\
{[\mathrm{meV}]}\end{array}$ & $\begin{array}{l}\mathbf{T}_{\text {iso }} \\
{[\mathrm{K}]}\end{array}$ & $\begin{array}{l}\mathbf{k}_{\mathbf{B}} \mathbf{T}_{\text {iso }} \\
{[\mathrm{meV}]}\end{array}$ & $\begin{array}{l}\mathbf{E}_{\mathbf{g}} \\
{[\mathrm{meV}]}\end{array}$ & $\begin{array}{l}\boldsymbol{W}_{\mathbf{h}} \\
{[\mathrm{meV}]}\end{array}$ \\
\hline $\mathrm{h}^{+}$ & 7.88 & 0.52 & 300 & 25.7 & 533 & 46 & 250 & 302 \\
\hline
\end{tabular}
dom walk from site to site ${ }^{[40]}$ with the hopping mobility

$$
\mu=\mu_{0} \cdot \mathrm{e}^{\frac{-W_{h}}{\mathrm{k}_{\mathrm{B}} \cdot \mathrm{T}}}
$$

where $W_{h}$ is the activation energy for hole polaron hopping from one lattice site to a neighbouring site. As discussed below, the

Table 1. The parameters $\mathcal{H}$ and $n$, activation energies and isokinetic temperatures for the determination of transport for holes $\mathrm{h}^{+}$ in the perovskite materials LSF. 
most plausible expression for $W_{h}$ is similar to that for $E_{\mathrm{g}}$ (Eqn. (13)):

$$
W_{h}=W_{m}-W_{n} \cdot n
$$

decreasing with $n$ from its maximum value, $W_{m}$. For the sample, $\mathrm{La}_{0.7} \mathrm{Sr}_{0.3} \mathrm{FeO}_{3}, \mathrm{n}=0.52,{ }^{[14]}$ and the polaron activation energy is $302 \mathrm{meV}$ according to Jung. ${ }^{[6]}$ By dielectric relaxation experiments, the polaron hopping energy can be determined to a good approximation when the disorder energy ${ }^{[41]}$ (the energetic disorder experienced by hopping carriers), which is typically very small, ${ }^{[42]}$ is neglected. For $\mathrm{La}_{(1-x)} \mathrm{Sr}_{\mathrm{x}} \mathrm{FeO}_{3}$ with $\mathrm{x}=0.05$ to 0.30 , Jung and Iguchi report ${ }^{[41]}$ activation energies from relaxation of $490 \mathrm{meV}$ to $230 \mathrm{meV}$, while the total activation energies range from $530 \mathrm{meV}$ to $290 \mathrm{meV}$, when $\mathrm{x}$ increases. With increasing $\mathrm{Sr}^{2+}$ substitution, $\mathrm{La}_{1-\mathrm{x}} \mathrm{Sr}_{\mathrm{x}} \mathrm{FeO}_{3}$ becomes increasingly covalent, ${ }^{[41]}$ and charge transport by polarons becomes less relevant. The maximum polaron hopping activation energy, for $n=0$, thus ranges by linear extrapolation to around $W_{h}=540 \mathrm{meV}$, and $0 \mathrm{meV}$ at $n=1$, so that we can estimate in linear approximation for Eqn. (15) $W_{h}$ $=540 \mathrm{meV}-540 \mathrm{meV} \cdot n$.

Considering the MNR and the isothermal temperature $\mathrm{T}_{\text {iso, }}$, we obtain

$$
\sigma(\mathrm{T})=\mathrm{n}_{\mathrm{h}^{+}}(\mathrm{T}) \cdot \mathrm{h}^{+} \cdot \mu(\mathrm{T})=\sigma_{00} \cdot \mathrm{e}^{\left(\frac{\Delta \mathrm{E}}{\mathrm{k}_{\mathrm{B}} \cdot \mathrm{T}_{\text {iso }}}\right)} \cdot \mathrm{e}^{\left(\frac{-\mathrm{Eg}_{\mathrm{g}}-\mathrm{W}_{h}}{\mathrm{k}_{\mathrm{B}} \cdot \mathrm{T}}\right)}
$$

The exponent, $\Delta \mathrm{E} / \mathrm{k}_{\mathrm{B}} \mathrm{T}_{\text {iso }}$, in Eqn. (16) is the MEE term. The term $\Delta \mathrm{E}$ may be the total activation energy, as in Eqn. (13), it may be $E_{F}-E_{V}$, or it may be $W$, depending upon whether they satisfy the condition of being 'large'. As we shall see, in the present case, that it is the total activation energy, as both components are large.

In the context of the preceding discussion, we are now in a position to find the meaning of the observations reported previously, and presented in Eqns (3) and (4). ${ }^{[14,15]}$ Eqn. (3) concerns only those terms in Eqn. (16) which vary with $n$. That is,

$$
\mathcal{H}=\left(\mathrm{E}_{\mathrm{g}}^{\prime}+\mathrm{W}_{\mathrm{n}}\right) \cdot\left(\frac{1}{\mathrm{k}_{\mathrm{B}} \mathrm{T}_{\mathrm{m}}}-\frac{1}{\mathrm{k}_{\mathrm{B}} \mathrm{T}_{\text {iso }}}\right)
$$

where $\mathrm{T}_{\mathrm{m}}$ is the temperature at which $\mathcal{H}$ is measured, and

$$
\sigma^{\prime}=\sigma_{00} \cdot \mathrm{e}^{\left(\mathrm{E}_{\mathrm{g}}^{0}+\mathrm{W}_{\mathrm{m}}\right) \cdot\left(\frac{1}{\mathrm{k}_{\mathrm{B}} \mathrm{T}_{\text {iso }}}-\frac{1}{\mathrm{k}_{\mathrm{B}} \mathrm{T}_{\mathrm{m}}}\right)}
$$

From the conductivity of LSF, data in ref. [43] we estimate the isokinetic temperature of LSF to be $\mathrm{T}_{\mathrm{iso}} \approx 533 \mathrm{~K}$, which yields $\mathrm{k}_{\mathrm{B}} \cdot \mathrm{T}_{\text {iso }}=46 \mathrm{meV}$. For the results cited in the previous section, $\mathrm{T}_{\mathrm{m}}$ was about $300 \mathrm{~K}$, which yields a thermal energy of $26 \mathrm{meV} \cdot{ }^{[10]}$ Band gap energies are reported to range from $330 \mathrm{meV}$ to $50 \mathrm{meV}$ for $\mathrm{x}=0.15$ to 0.9 in $\mathrm{La}_{(1-\mathrm{x})} \mathrm{Sr}_{\mathrm{x}} \mathrm{FeO}_{3} \cdot{ }^{[39]}$

In linear approximation, this would yield $373 \mathrm{meV}$ for for $E^{\prime}$. For $W_{n}$ we estimated $W_{n}=540 \mathrm{meV}$. Then $W_{n}+E_{g}^{\prime}{ }_{g}^{o}$ is approximately 913 meV. With the data, which we have listed in Table 1, we can check for the validity of our considerations:

$$
\begin{gathered}
\mathcal{H}=\left(\mathrm{E}_{\mathrm{g}}^{\prime}+\mathrm{W}_{\mathrm{n}}\right) \cdot\left(\frac{1}{\mathrm{k}_{\mathrm{B}} \mathrm{T}_{\mathrm{m}}}-\frac{1}{\mathrm{k}_{\mathrm{B}} \mathrm{T}_{\mathrm{iso}}}\right) \\
=(373 \mathrm{meV}+540 \mathrm{meV}) \cdot\left(\frac{1}{26 \mathrm{meV}}-\frac{1}{46 \mathrm{meV}}\right) \cong 14
\end{gathered}
$$

which is less than a factor two larger than the experimentally determined value for $\mathcal{H}=7.88$ in ref. [14]. This is plausible and acceptable.

What is the physical origin of the dependence of $W$ on $n$ ? To our knowledge, there is only one model for polaronic conductors which predicts a dependence of any kind; it predicts sublinear dependence. The correlated barrier hopping $(\mathrm{CBH})$ model has been used principally to explain the behavior of amorphous chalcogenides. It was first proposed by Pike ${ }^{[4]}$ and developed by Elliott ${ }^{[45,46]}$ for AC conductivity, applied to DC conductivity by Pramanik, ${ }^{[47]}$ and modified by Abdel-Wahab ${ }^{[48,49]}$ to take account of MNR. The model is founded on three assumptions. First, an isolated trap can have a very high barrier to trapping and to detrapping $W_{m}$. Second, when two traps are close together in distance $r$, their ${ }^{m}$ Coulomb potentials can lower the barrier between them:

$$
W_{h}=W_{m}-\frac{2 \cdot e^{2}}{\pi \cdot \varepsilon \cdot r}
$$

Finally, the distances $r$ of the traps are randomly distributed. Then, the distance relevant for DC conductivity is the percolation distance $r_{p}$, so that

$$
W_{h}\left(\mathrm{r}_{\mathrm{p}}\right)=W_{m}-\frac{2 \cdot \mathrm{e}^{2}}{\pi \cdot \varepsilon \cdot \mathrm{r}_{\mathrm{p}}}
$$

The percolation distance $r_{p}$ scales with $\mathrm{N}^{-1 / 3}$ where $\mathrm{N}$ is the volume density of traps:

$$
r_{p}=\left(2.7 \cdot \frac{3}{4 \cdot \pi \cdot N}\right)^{1 / 3}
$$

The $\mathrm{CBH}$ model reproduces charge transport in amorphous chalcogenides quite well. In particular, the form which incorporates MNR permits fitting of the temperature dependence of ac conductivity over a wide range, ${ }^{[45]}$ fitting the frequency dependence of AC conduction, ${ }^{[48]}$ and describing $\mathrm{AC}$ and DC conductivity with the same parameters, ${ }^{[50]}$ It also predicts that the isokinetic and high field, 'Poole-Frenkel' temperatures should be the same, as observed in one case. ${ }^{[34]}$ But the form used for the chalcogenides is clearly in contradiction with the observation of a linear dependence of the hole conductivity in perovskites in so far as the hole concentration, $n$, is determined by the doping fraction, $x$.

We suggest that the model may be made compatible with experiment if we assume that conductivity in perovskites involves only movement of carriers in the channels in the structure, that traps outside of a channel have negligible effect on carrier trapping inside the channel, and that the traps in a given channel are randomly distributed. Then, the resulting one-dimensional model will yield a linear dependence. This requires further study.

\subsection{Proton Conductivity}

In the case of proton polarons in perovskites, the protons are part of the crystal structure of the material, before they become charge carriers. ${ }^{[16,17]}$ The proton conductor has the stoichiometry $\mathrm{ABO}_{3-\delta}$, and the $\delta$ accounts for the oxygen vacancies. For example, in $\mathrm{BaCe}_{(1-\mathrm{x})} \mathrm{Y}_{\mathrm{x}} \mathrm{O}_{3}$, the charge carrier concentration $\mathrm{n}$ depends upon the substitution level by $\mathrm{Y}^{3+}$, which determines $\delta$. The vacancies are filled with the oxygen ions of $\mathrm{H}_{2} \mathrm{O}$ molecules when the material is exposed to water vapor. The two hydrogen ions from the water molecule, i.e. protons will form $\mathrm{OH}^{-}$bonds with adjacent oxygen ions in the lattice. Only upon heating of this water-loaded perovskite, will the hydroxyl bonds 
melt. ${ }^{[18,51]}$ The melting of these bonds coincides with the onset of the proton conductivity at a characteristic temperature range, which is around $650 \mathrm{~K}$ for the aforementioned stoichiometry. This is illustrated in Fig. 4, where the proton diffusion constant $\mathrm{D}$ in $\mathrm{BaZr}_{0.9} \mathrm{Y}_{0.1} \mathrm{O}_{2.95}$ is plotted versus the temperature $\mathrm{T}$. In the same figure, we have plotted the relative thermal expansion coefficient $\alpha$ for the 'dry' and for 'hydrated' material, which we obtained from high-resolution neutron diffraction data. When dry BZY becomes humidified, the thermal expansion coefficient increases from 6.19 to $6.45 \times 10^{-6} \mathrm{~K}^{-1}$. At around the onset temperature for proton conductivity around $600 \mathrm{~K}$ to $700 \mathrm{~K}$ we notice, for the hydrated sample, that $\alpha$ decreases from 6.45 to $4.56 \times 10^{-6} \mathrm{~K}^{-1}$

The proton charge carriers are formed by exposing the ceramic proton conductor to humidity, which then absorbs the $\mathrm{H}_{2} \mathrm{O}$ molecules. The water molecules become split by thermal activation. We can estimate the necessary energy from data, which, for example, were published by Ricote et al. ${ }^{[52]}$ for $\mathrm{BaCe}_{(0.9-x)} \mathrm{Zr}_{\mathrm{x}} \mathrm{Y}_{0.1} \mathrm{O}_{(3-\delta)}$. For temperatures above $800 \mathrm{~K}$, we obtain from an Arrhenius plot of the data of Fig. 7 in ref. [52] proton formation energies of around $E_{p}=-200 \mathrm{meV}$. The proton concentration decreases with increasing temperature. This decrease is relatively small so that the number of carriers may be treated as constant, or using a small correction.

In a study[53] of $\mathrm{BaZr}_{(1-\mathrm{x})} \mathrm{Y}_{\mathrm{x}} \mathrm{O}_{3}$, it was found that the formal proton concentration scales linearly with Y-dopant concentration. However, only $1 / 3$ of the $\mathrm{x}$ sites are filled with the $\mathrm{OH}$ - groups and thus with the protons. Proton concentration increases sigmoidally with water partial pressure $\mathrm{p}_{\mathrm{H} 2 \mathrm{O}}$. At $500{ }^{\circ} \mathrm{C}$ and at $400{ }^{\circ} \mathrm{C}$, conductivity exhibits a small, linear, increase with increasing water partial pressure and thus, with increasing proton concentration. At $600{ }^{\circ} \mathrm{C}$, there is a linear decrease in conductivity with increasing water pressure, and thus, with increasing proton concentration (compare Fig. 4 in the study by Bonanos ${ }^{[54]}$ ).

For $\mathrm{LaMnO}_{3}, E_{\mathrm{p}}$, the activation energy for $n$, the analogue of $E_{\mathrm{g}}$, was found to be $86 \mathrm{meV} \cdot{ }^{[55]}$ For $\mathrm{La}_{(1-\mathrm{x})} \mathrm{Sr}_{\mathrm{x}} \mathrm{FeO}_{3}$, it may range from 40 to $80 \mathrm{meV},{ }^{[41]}$ depending upon the stoichiometry parameter $\mathrm{x}$. That is, it appears that $E_{\mathrm{p}}$ is small (or nil) for perovskites, and will not exhibit MNR behavior. In contrast, for all three perovskites discussed above, the mobility of protons $p^{+}$ is thermally activated

$$
\mu(T)=\mu_{0} \cdot e^{-\frac{\Delta E}{k_{B} T}}
$$

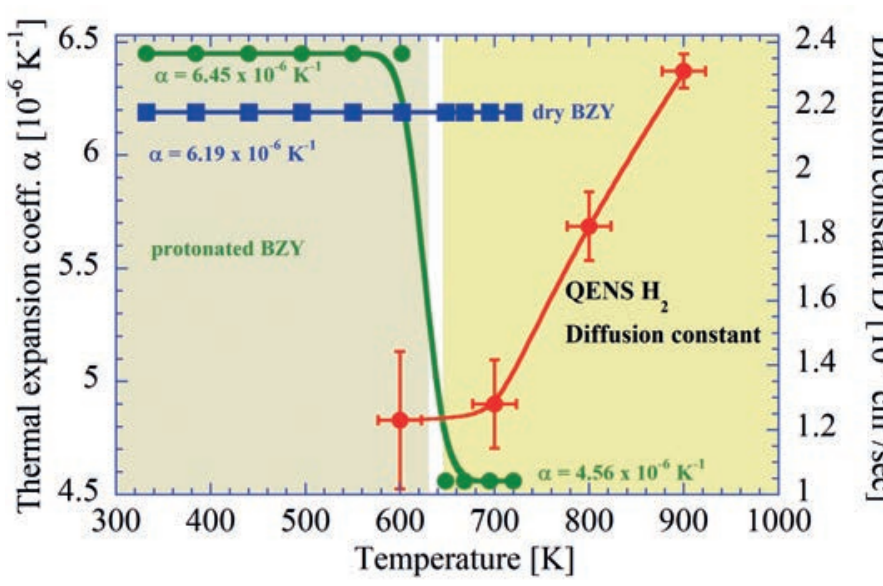

Fig. 4: Variation of the thermal expansion coefficient of $\mathrm{BaZr}_{0.9} \mathrm{Y}_{0.1} \mathrm{O}_{2.95}$ when dry (blue squares) and when protonated in water vapour (green circles) with sample temperature, along with the variation of the proton diffusivity as measured with quasi-elastic neutron scattering (red bullets). Solid lines are guides for the eyes. where

$$
\mu_{0}=\mu_{0}(T)=\frac{D_{0} \cdot p^{+}}{k_{B} \cdot T}
$$

and $\Delta E$ is expected to be large. Nevertheless, unlike the case of hole polarons, we find from the data in ref. [53] that proton conductivity increases linearly with the number of carriers, and so, with the number of traps. Specifically, we find no indication for an exponential relation between proton conductivity and the proton concentration in perovskites. Hence, Eqn. (3) and the formalism, which we developed from it for the hole conductivity, seems not to be applicable for proton conductivity. Further fundamental work will be necessary for finding a universal description for both transport processes.

For protons in minerals, which are known to be polaronic, $[37,56]$ Jones ${ }^{[56]}$ found that the hopping activation energy for diffusion decreases as the $1 / 3$ power of the humidity content. This suggests that protons in minerals obey the three-dimensional $\mathrm{CBH}$ model.

\section{Conclusions}

We have studied the electronic and protonic conductivities of metal oxides with perovskite structure, which are considered for use as electrodes and solid electrolytes in fuel cells. While they are used as different components, their function has striking similarities, which manifests in thermally activated dynamics of holes and protons as relevant charge carriers. The underlying Arrhenius plots show a typical Meyer-Neldel Rule characteristic, at least for a limited stoichiometry range. The experimentally determined activation energies and charge carrier concentrations are the empirical parameters, along with the temperature, which can be set in relation with a newly introduced quantity $\mathcal{H}$ for hole conductors, which scales exponentially with the DC conductivity. The isothermal temperature, as determined from the MNR and the temperature at measurement, yield thermal energies, by which the aforementioned activation energies are weighed, so as to arrive at the product of parameter $\mathcal{H}$ and charge carrier concentration $n$. The relevant activation energies in this respect are the polaron hopping activation energy, and the thermal energy necessary to generate charge carriers from the band gap of semiconducting electrode materials. This model finds its limits where the electronic structure changes from ionic bonding to covalent bonding. High activation energy kinetics calls for the inclusion of a multi-excitation entropy (MEE) term, which is fixed to the isokinetic temperature of the MNR. Consideration of this MEE term in the numerical evaluation of the activation energies for the charge carrier generation and polaron hopping, only, for the hole polaron, and not for the proton polaron, shows that the sum of activation energies of either system, weighed by the difference of the thermal energies for the sample environment temperature and isokinetic temperature, yields values which are comparable between the theoretical model and experimental data for the hole transport. However, for the proton polarons, the conductivity increases linearly with the charge carrier proton concentration, and thus the mathematical formulation deserves further exploration.

\section{Acknowledgements}

The neutron data shown in this paper were recorded at the Swiss Neutron Spallation Source (SINQ) and analyzed by Dr. Alejandro Ovalle (Empa), and Dr. Vladimir Pomjakushin and Dr. Jan Embs, both PSI SINQ. During writing of this manuscript, A.B. had support from the Horizon 2020 FET-Flagship CSA project 'SUNRISE - Solar Energy for a Circular Economy', grant agreement $n^{\circ}$ 816336, and A.B. and Q.C. had support from the Swiss National Science Foundation project 'Proton conductivity in epitaxial strained thin films', project no ${ }^{\circ} 200021-188588$, Q.C. had support from the National Nature Science Foundation of China 
project 'Effect of strain on the proton polaron transport in perovskitetype proton conducting epitaxial films', project no ${ }^{\circ} 51802193$.

Received: October 16, 2019

[1] R. Jost, in 'Das Maerchen vom Elfenbeinernen Turm', Vol. 34, Eds. K. Hepp, W. Hunziker, W. Kohn, Springer, Berlin, Heidelberg, 1995, p. 183.

[2] S. Nishijima, S. Eckroad, A. Marian, K. Choi, W. S. Kim, M. Terai, Z. Deng, J. Zheng, J. Wang, K. Umemoto, J. Du, P. Febvre, S. Keenan, O. Mukhanov, L. D. Cooley, C. P. Foley, W. V. Hassenzahl, M. Izumi, Supercond. Sci. Tech. 2013, 26, 113001 .

[3] K. D. Kreuer, Chem. Mater. 1996, 8, 610

[4] N. Rosch, Chem. Phys. 1973, 1, 220.

[5] Y. A. Firsov, Semiconductors 1995, 29, 515

[6] W. H. Jung, Physica B 2001, 299, 120.

[7] W. H. Jung, Mater. Lett. 2002, 57, 697.

[8] W.-H. Jung, J. Mater. Sci. 2006, 41, 3143 .

[9] W.-H. Jung, Mater. Lett. 2007, 61, 2274.

[10] L. D. Landau, S. I. Pekar, Zh. Eksp. Teor. Fiz. 1948, 18, 419.

[11] A. Braun, B. S. Mun, Y. Sun, Z. Liu, O. Gröning, R. Mäder, S. Erat, X. Zhang, S. S. Mao, E. Pomjakushina, K. Conder, T. Graule, J. Electron Spectrosc. 2010, 181, 56.

[12] A. Braun, J. Richter, A. S. Harvey, S. Erat, A. Infortuna, A. Frei, E. Pomjakushina, B. S. Mun, P. Holtappels, U. Vogt, K. Conder, L. J. Gauckler, T. Graule, Appl. Phys. Lett. 2008, 93, 262103.

[13] A. Braun, 'X-ray Studies on Electrochemical Systems - Synchrotron Methods for Energy Materials', Walter De Gruyter GmbH, Berlin/Boston, 2017.

[14] A. Braun, D. Bayraktar, S. Erat, A. S. Harvey, D. Beckel, J. A. Purton, P. Holtappels, L. J. Gauckler, T. Graule, Appl. Phys. Lett. 2009, 94, 202102.

[15] A. Braun, S. Erat, D. Bayraktar, A. Harvey, T. Graule, Chem. Mater. 2012, $24,1529$.

[16] F. Fillaux, H. Ouboumour, C. H. Cachet, J. Tomkinson, C. Lévy-Clément, L. T. Yu, J Electrochem. Soc. 1993, 140, 592.

[17] F. Fillaux, H. Ouboumour, J. Tomkinson, L. T. Yu, Chem. Phys. 1991, 149, 459.

[18] Q. Chen, F. El Gabaly, F. A. Akgul, Z. Liu, B. S. Mun, S. Yamaguchi, A. Braun, Chem. Mater. 2013, 25, 4690.

[19] A. Braun, Q. Chen, Nat. Commun. 2017, 8, 15830.

[20] A. L. Samgin, Solid State Ion. 2000, 136, 291.

[21] A. Braun, S. Duval, P. Ried, J. Embs, F. Juranyi, T. Straessle, U. Stimming, R. Hempelmann, P. Holtappels, T. Graule, J. Appl. Electrochem. 2009, 39, 471.
[22] B. Groß, C. Beck, F. Meyer, T. Krajewski, R. Hempelmann, H. Altgeld, Solid State Ion. 2001, 145, 325

[23] S. Glasstone, E. F. Hammel, J. Am. Chem. Soc. 1941, 63, 2003.

[24] A. Yelon, B. Movaghar, R. S. Crandall, Rep. Prog. Phys. 2006, 69, 1145.

[25] F. H. Constable, Proc. Roy. Soc. A 1925, 108, 355.

[26] A. Yelon, B. Movaghar, H. M. Branz, Phys. Rev. B 1992, 46, 12244.

[27] W. Meyer, H. Neldel, Phys. Z. 1937, 38, 1014.

[28] D. Emin, Phys. Rev. Lett. 1974, 32, 303.

[29] D. Emin, Adv. Phys. 1975, 24, 305.

[30] A. Yelon, B. Movaghar, Phys. Rev. Lett. 1990, 65, 618

[31] F. Giannici, M. Shirpour, A. Longo, A. Martorana, R. Merkle, J. Maier, Chem. Mater. 2011, 23, 2994.

[32] J. C. Wang, Y. F. Chen, Appl. Phys. Lett. 1998, 73, 948.

[33] K. Shimakawa, F. AbdelWahab, Appl. Phys. Lett. 1997, 70, 652.

[34] A. Yelon, B. Movaghar, Appl. Phys. Lett. 1997, 71, 3549.

[35] F. Abdel-Wahab, A. Yelon, J. Appl. Phys. 2013, 114, 6

[36] A. G. Jones, Geochem. Geophy. Geosy. 2014, 15, 337.

[37] A. Yelon, MRS Adv. 2017, 2, 425.

[38] A. S. Alexandrov, J. T. Devreese, 'Advances in Polaron Physics', Springer, Springer Berlin Heidelberg, 2010.

[39] Y. J. Xie, M. D. Scafetta, E. J. Moon, A. L. Krick, R. J. Sichel-Tissot, S. J. May, Appl. Phys. Lett. 2014, 105, 062110.

[40] T. Holstein, Ann. Phys. 1959, 8, 343

[41] W. H. Jung, E. Iguchi, J. Phys.-Cond. Matt. 1995, 7, 1215.

[42] L. A. K. Dominik, R. K. MacCrone, Phys. Rev. 1967, 163, 756.

[43] J. Matsuno, T. Mizokawa, A. Fujimori, K. Mamiya, Y. Takeda, S. Kawasaki, M. Takano, Phys. Rev. B 1999, 60, 4605.

[44] G. E. Pike, Phys. Rev. B 1972, 6, 1572.

[45] S. R. Elliott, Philos. Mag. 1977, 36, 1291.

[46] S. R. Elliott, Adv. Phys. 1987, 36, 135.

[47] M. H. A. Pramanik, P. N. Butcher, I. D. Cox, Philos. Mag. B 2012, 47, 437.

[48] F. Abdel-Wahab, J. Appl. Phys. 2002, 91, 265.

[49] F. Abdel-Wahab, Philos. Mag. B 2009, 82, 1327.

[50] F. Abdel-Wahab, A. A. Montaser, A. Yelon, Monatsh. Chem. 2012, 144, 83.

[51] A. Braun, A. Ovalle, V. Pomjakushin, A. Cervellino, S. Erat, W. C. Stolte, T. Graule, Appl. Phys. Lett. 2009, 95, 224103.

[52] S. Ricote, N. Bonanos, G. Caboche, Solid State Ion. 2009, 180, 990.

[53] S. B. C. Duval, PhD Thesis, Technische Universität München 636082, München, 2008.

[54] N. Bonanos, Solid State Ion. 2001, 145, 265.

[55] W.-H. Jung, J. Mater. Sci. 2002, 21, 573.

[56] A. G. Jones, Geochem. Geophy. Geosy. 2014, 15, 2616. 